\title{
Resource management issues for multi-carrier relay-enhanced systems
}

\author{
Jacek Góra ${ }^{1,2^{*}}$ and Simone Redana ${ }^{3}$
}

\begin{abstract}
Relaying is one of the enabling techniques for next generation systems. Factors deciding on performance of relayenhanced systems are the resource allocation and coordination. The capacity of the wireless relay backhaul link is typically the bottleneck of transmissions conveyed through the relay node. It can be to some extent improved by allocating dedicated resources; however, typically this is done at the expense of the performance of users served directly from the base station. To achieve optimum overall performance, resource assignment to relays and users has to be done carefully. This article addresses the problem of resource allocation with relays operated in multicarrier scenarios. Guidelines for optimization of the resource allocation under the resource fair policy are given with different relay configurations, including time, frequency, and hybrid resource partitioning schemes. Finally, advanced resource coordination procedures are presented. Multi-carrier interference coordination is proposed for improving the quality of radio links, and carrier load balancing for improving the efficiency of resource utilization.
\end{abstract}

Keywords: LTE-advanced, relay nodes, radio resource management, multi-carrier

\section{Introduction}

The concept of a three-node communication channel was first proposed by van der Meulen [1]. In his article, van der Meulen introduces an additional node to a traditional two-node communication channel. The purpose of the new node (in the following we refer to it as the relay node, $\mathrm{RN}$ ) is to support communication between the source and the target nodes. Later, this concept has been further developed [2,3] and proposed for application in radio communication networks [4].

Currently, relaying is a mature technique envisioned mainly as a cost-efficient solution for extending coverage of cellular networks, improving cell-edge performance and in some cases also enhancing overall cell capacity [5-9]. As such, relaying is being included in standards of next generation cellular systems. The two main systems supporting relaying are the Long-Term Evolution-Advanced (LTE-Advanced) [10] from the 3rd Generation Partnership Project (3GPP) forum and the mobile Worldwide Interoperability for Microwave Access (WiMAX) standard IEEE 802.16 m [11,12].

\footnotetext{
* Correspondence: jacek.gora@nsn.com

${ }^{1}$ Nokia Siemens Networks - Research - Radio Systems, ul. Strzegomska 2-4,

53-611 Wroclaw, Poland

Full list of author information is available at the end of the article
}

Over the years, many concepts of the RN functionality have been proposed. They can be divided into two main groups: cooperative and non-cooperative relaying. In the cooperative relaying scheme, both the source and the RN communicate with the target node. Many solutions have been proposed for this cooperation, mainly based on different coding schemes such as network coding [13], space-time coding [14], etc. A comprehensive analysis of different cooperative relaying concepts can be found in [15]. Some of the recent studies on the topic are also presented in [16].

The second approach to relaying is the non-cooperative scheme. In this concept, the target node communicates only with the relay node, which then forwards the received data from the source node to the target node. This communication scheme is also called the multihop transmission. Mainly for practical implementation reasons the non-cooperative relaying is the one currently considered for commercial applications $[17,18]$.

The current 3GPP LTE Release-10 standard defines $\mathrm{RN}$ as a dedicated node communicating bidirectionally with a macro base station (donor eNodeB, DeNB) on one side and a user equipment (UE) on the other side (Figure 1). The DeNB-RN link is called the wireless backhaul link and the RN-UE link is called the access 


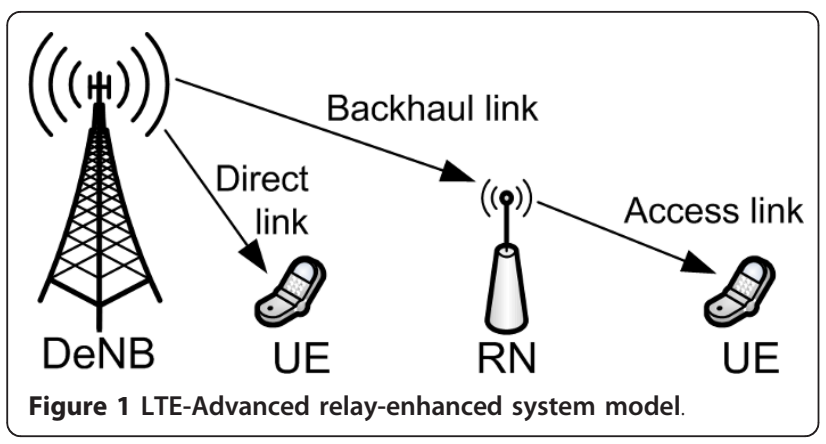

link. Depending on whether the UE is aware of the RN existence or not, the RN can be characterized as either non-transparent (Type-1) or transparent (Type-2), respectively [16]. In the LTE-Advanced Release-10 specification the Type-1 relays have been prioritized [17]. In this configuration, the $\mathrm{RN}$ creates its own cell and from the UE perspective is perceived as a base station.

In both communication directions (downlink and uplink), the RN performs a full reception of incoming signals, including demodulation and decoding. The data received from the source node is temporarily stored in a buffer and then retransmitted to the target node. This mode of operation is called decode-and-forward (DF) (studied and compared with other relaying modes, e.g., [18-21]). By making full reception, the DF RN is able to regenerate the transmission, i.e., remove interference and correct errors. It is also able to change the modulation and coding scheme (MCS) to adapt it to the quality of the link towards the target node. Due to this processing, however, a retransmission delay is introduced.

Because of the retransmission delay and potentially changed format, the transmission incoming and outgoing from a DF RN is different at each time instance. This creates the need to separate the two links for cross-interference protection. Without the sufficient separation interference from the relay-target link would significantly decrease quality of the source-relay link (the so-called loop or self-interference explicitly addressed, e.g., [22-24]).

As defined by the 3GPP, the separation of the wireless backhaul and access links can be provided by either sufficient shielding and separation in space between the backhaul and access link antennas (Type-1b) or by resource partitioning, i.e., operating the two links on different radio resources. The backhaul-access link resource partitioning can be done in time domain (basic Type-1, in-band) or in frequency domain (Type-1a, outband). As the shielding and in-space separation are applicable only in specific cases, e.g., an indoor RN with an outdoor backhaul link antenna, the resource partitioning-based solutions are typically considered.
Deployment of RNs involving resource partitioning imposes the need for a precise control of the amount of resources assigned to each link. The problem of an optimum resource partitioning was in the past typically studied for single-carrier scenarios. The authors of $[25,26]$ discuss and compare several resource sharing techniques for in-band RNs, while in [27] the focus is on the resource split at DeNB between RNs and directly served UEs. Further, in $[28,29]$ the authors consider radio resource management solutions for RNs involving also elements of interference coordination and offloading aspects.

In this article, resource management for multi-carrier relay-enhanced networks is discussed. The multi-carrier operation is aside relaying one of the key solutions included in standards of next generation systems [30-33]. Considering this, the combination of relaying and multi-carrier operation is a probable use case in the evolution of LTE-Advanced, and it should be explicitly studied. The availability of multiple carriers enables the frequency domain resource partitioning along with advanced coordination solutions that can be implemented to improve performance of relay-enhanced systems. All these aspects with example solutions are discussed in this article.

The rest of the article is organized as follows. Section 2 presents models of cellular systems with and without relay nodes. For both cases, quality of radio links is assessed and discussed with a focus on achieving increased performance fairness across the network. In Section 3, aspects of efficient resource assignment for RNs and UEs are discussed. Criteria for an optimal resource management under the resource fair (RF) assignment policy are specified, and different resource partitioning schemes (in-band, out-band, and hybrid) are discussed and compared with respect to those criteria. In Section 4, advanced solutions for coordinated resource management including carrier load balancing and interference coordination are presented. Finally, Section 5 concludes findings of this article. All the evaluations presented in this article are done according to the methodology described in the Appendix.

\section{System model and problem definition}

In Section 2.1, we derive the distribution of the UE spectral efficiency (SE) in the network and in Section 2.2, we show how it can be improved by RN deployment.

\subsection{Relay-less system model and its shortcomings}

One of the main goals of a cellular system is to enable user mobility with constant connectivity. This is provided by deploying multitude of base stations, each supporting network coverage on a limited area-referred to as a cell. As a user moves across the network, its 
connection is being served by different base stations. For the selection of the most suitable serving station, the UE continuously measures and compares signal strengths of nearby base stations. Typically, selection of the serving station is based on the highest received signal power criteria $[34,35]$.

Quality of the radio link between the UE $u$ and its serving base station $j_{0}$ is commonly characterized with the signal-to-interference and noise ratio (SINR) [36]

$$
\operatorname{SINR}\left(j_{0}, u\right)=\frac{P_{R x}\left(j_{0}, u\right)}{P_{\mathrm{Th}}+\sum_{j \neq j_{0}} P_{R x}(j, u)}
$$

where $P_{\mathrm{Rx}}(j, u)$ is the power of signal of the base station $j$ received by the user $u$ and $P_{\text {Th }}$ is the thermal noise power.

The quality of the link can also be characterized in terms of SE, i.e., the maximum data rate possible to be achieved with the given SINR per unitary channel bandwidth. The SE is commonly estimated based on the Shannon formula [36,37].

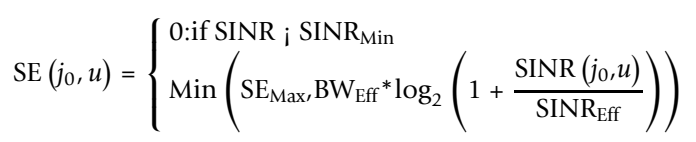

where SINR $_{\text {Min }}$ is the minimum SINR enabling correct signal reception, $\mathrm{SE}_{\mathrm{Max}}$ is the maximum $\mathrm{SE}$ related to the most effective MCS available in the system, $\mathrm{BW}_{\mathrm{Eff}}$ is the bandwidth efficiency accounting for such factors as guard bands, control channel overhead, etc., and SINR Eff $_{\text {f }}$ is the SINR efficiency related to the efficiency of link adaptation, transmitter and receiver implementation, etc. In this study, $2 \times 2$ MIMO transmission is considered with optimal switching between dual-stream and beamforming transmission schemes. For such configuration, the following Shannon formula fitting parameters are considered: SINR $_{\text {Min }}=-10 \mathrm{~dB}, \mathrm{BW}_{\text {eff }}=0.87, \mathrm{SINR}_{\text {eff }}$ $=1.08$, and $\mathrm{SE}_{\mathrm{Max}}=8 \mathrm{bit} / \mathrm{s} / \mathrm{Hz}$.

The SE metric is especially useful, as it enables direct estimation of the data rate available on the link given by

$$
T\left(j_{0}, u\right)=R * S(u) * \operatorname{SE}\left(j_{0}, u\right)
$$

where $T\left(j_{0}, u\right)$ is the data rate (throughput) of the user $u$ at the base station $j_{0}, R$ is the available system bandwidth and $S(u)$ is the fraction of the base station resources available for the UE $u$ according to the selected resource assignment policy.

The most common resource assignment policy is the RF. An example of this policy is the round-robin scheduling. With this approach, all UEs served by one base station are assigned in long term with an equal amount of resources, which is

$$
S(u)=N^{-1}
$$

where $N$ is the number of active UEs connected to the base station.

A common problem of cellular networks is the nonuniformity of performance across the cell area. Explicitly, radio conditions available for UEs located close to the base station (cell center) are significantly better than the conditions observed by the UEs at the cell edge. The non-uniformity of radio conditions observed by UEs in different locations in a suburban scenario $(1732 \mathrm{~m}$ inter-site distance between base stations) is illustrated in Figure 2, where cumulative distribution function of the SE values collected over the whole network area is presented. More details on the assumed scenario can be found in Appendix.

Specifically, it can be estimated that with the RF assignment policy approximately $2 / 3$ of all UEs achieve no more than $1 / 3$ of the maximum achievable data rates. By using Jain's index (5) [38], the uniformity score for SE can be calculated as 0.64 (0-to- 1 scale, with 1 in case of perfect uniformity).

$$
J(x)=\frac{E(x)^{2}}{E\left(x^{2}\right)}=\frac{E(x)^{2}}{E(x)^{2}+V(x)}
$$

where $J(x)$ is the Jain's index of variable $x, E(x)$ is its expected value and $V(x)$ is its variance.

\section{2. Relay-enhanced system model}

One solution available to improve the uniformity of radio conditions in a system is the deployment of RNs in the areas of poor connection quality towards macro base stations. RNs deployed in those locations attract nearby UEs by providing them improved radio conditions. At the same time the RNs should also have better

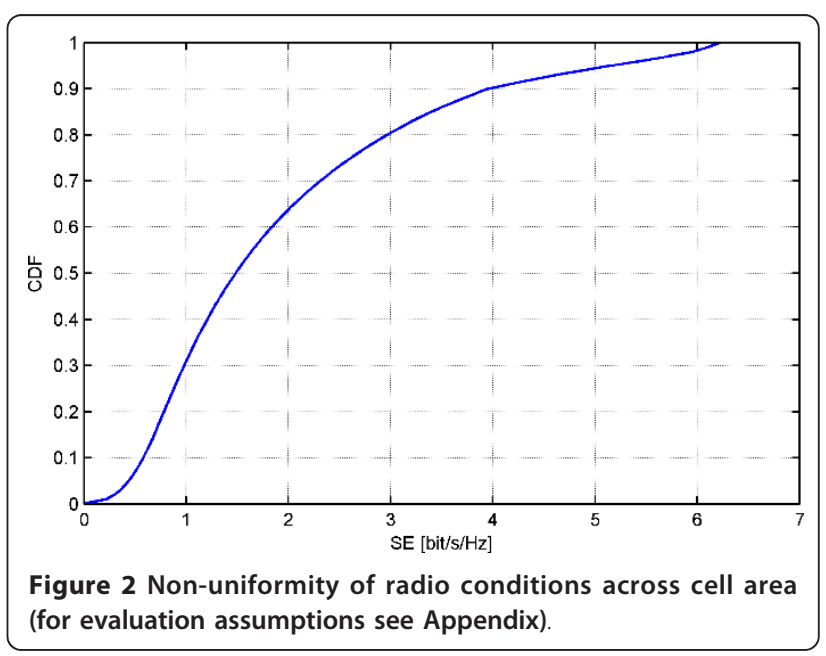


radio conditions towards the macro base station on the wireless backhaul link than typically observed by UEs. The improvement of the wireless backhaul link quality compared to the direct DeNB-UE link may come, e.g., from

- No penetration loss when UEs are indoors and the RN backhaul link antenna is outdoors;

- High elevation of RN antennas, thus better propagation conditions compared to the DeNB-UE link (this is especially valid in coverage limited scenarios, where signal power from DeNB measured by a UE is at the noise level or below);

- RN site planning resulting in higher probability of line-of-sight (LOS) connection and better SINR with the serving DeNB than with other base stations (e.g., as discussed in [39]);

- Directional antenna for communication on the backhaul link suppressing some of the interference from other base stations.

Quality of the backhaul link towards the serving DeNB $j_{0}$, considering the listed factors, can be expressed as

$$
\operatorname{SINR}_{\mathrm{BH}}\left(j_{0}, r\right)=\frac{P_{R x}\left(j_{0}, u_{r}\right) * G\left(j_{0}, r\right)}{P_{\mathrm{Th}}+\sum_{j \neq j_{0}} P_{R x}\left(j, u_{r}\right) * G(j, r) * a(j, r)}
$$

$$
\begin{aligned}
& \forall j \neq j_{0}: 1<G(j, r)<G\left(j_{0}, r\right) \\
& \forall j \neq j_{0}: a(j, r) \leq 1
\end{aligned}
$$

where $P_{\mathrm{Rx}}\left(j, u_{r}\right)$ is the signal power from the base station $j$ that would be received by a user $u_{r}$ placed in the position of RN $r, G(j, r)$ is the propagation gain on link between the eNB $j$ and the RN $r$ coming from the RN backhaul antenna elevation and site planning, and $a(j, r)$ is the level of signal suppression caused by the directional characteristics of the RN backhaul antenna.

The impact of deploying $10 \mathrm{RNs}$ along the edges of each DeNB cell in suburban scenario is analyzed next (details on the assumed scenario can be found in Appendix). As shown in Figure 3 the effects are twofold.

On one hand, performance of cell-edge UEs is significantly improved. SE of the RN-UE link is on average 83\% higher than the SE of the DeNB-UE link and the SE of the RN backhaul link is even higher (134\% higher than for the DeNB-UE link). As from the DeNB perspective an RN-connected UE is served with the SE of the RN backhaul link, this means that $\mathrm{RN}$-connected UEs need on average 2.34 times less resources compared to the situation when they are connected directly to the DeNB. Alternatively, if the RN-connected UEs use the same amount of DeNB resources (i.e., the resources that would be assigned to cell-edge UEs are assigned to RN
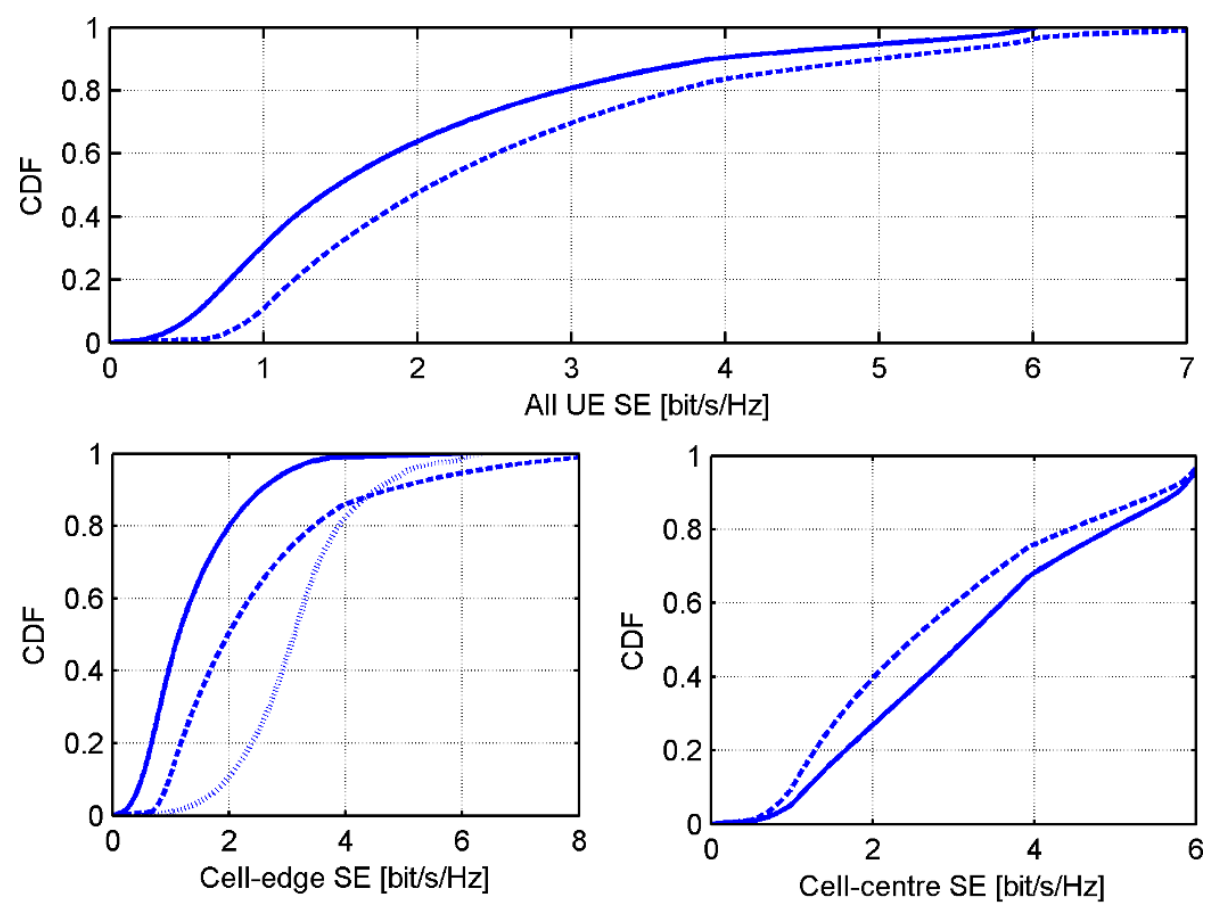

UE SE w/o RNs .--.---- UE SE with RNs

RN backhaul link SE

Figure 3 Improvement of radio conditions brought by RN deployment (for evaluation assumptions see Appendix). 
backhaul links), the achieved data rates are on average 2.34 times higher than they would be with direct DeNBUE connection.

On the other hand, the UEs still connected to the DeNB (cell-center UEs) experience additional interference from the RN side and therefore lower SE (on average $13 \%$ degradation) compared to the case of no RN deployment. However, the deployment of RNs offloads the DeNB (i.e., there is less UEs connected to DeNB after deployment of RNs and they require less resources to achieve better throughputs), thus the UEs still connected to the DeNB are expected to experience higher resource availability and therefore potentially higher throughputs despite the lower SE.

Overall, the deployment of RNs can improve performance for all UEs (DeNB- and RN-connected), the key factor is, however, proper control of the amount of resources assigned to the RN links. The resource assignment should take into account qualities of all links and the traffic offloading from DeNB to RNs.Resource management options considering the factors are discussed next in Sections 3 and 4.

\section{Resource partitioning in multi-carrier systems}

In a relay-enhanced system, three types of links coexist: direct (DeNB-UE), backhaul (DeNB-RN), and access (RN-UE). The purpose of the radio resource management procedures is to optimally assign system resources to the three link types in a way guaranteeing good performance for all UEs. To achieve that the three links need to be considered together as discussed in Section 3.1. In Section 3.2, the resource management is employed in the time and frequency domain resource partitioning.

\subsection{Resource allocation framework}

In relay-enhanced systems, we have that

- The same resources cannot be assigned to direct and backhaul links at one DeNB.

- The same resources cannot be assigned to the backhaul and access links of one RN.

- Access links of different RNs can reuse the same resources, but in such case inter- $\mathrm{RN}$ access-to-access link interference needs to be considered.

To ensure sufficient performance for all UEs in the system, i.e., those served directly from the DeNB and via the RNs, and to maximize efficiency of resource utilization the following guidelines should be considered:

(G1) An RN requires an amount of resources on the backhaul link that corresponds to its traffic offloading from the DeNB, i.e., the number and the service demand of the UEs attached to each RN should be considered in the resource assignment at the DeNB $[25,26]$.

(G2) Capacities available on the backhaul and access links of an RN should be equal in long-term average otherwise one of the two links would result to be the bottleneck with a waste of resource on the other link.

Considering the above-listed statements, the most efficient resource allocation schemes both at DeNB and RN are looked for next in the case of a system with $10 \mathrm{RNs}$ deployed per each DeNB's cell.

\subsubsection{Resource allocation at DeNB}

The guideline (G1) guarantees that every UE, irrespectively if it is connected to an $\mathrm{RN}$ or directly to the DeNB, has access to a fair amount of resources. For the RF allocation policy, the fraction of all DeNB resources assigned to a backhaul link can be defined as

$$
S_{\mathrm{BH}}(r)=\frac{N_{r}}{N_{d}+\sum_{r=1}^{N_{R}} N_{r}}=\frac{N_{r}}{N}
$$

where $N_{r}$ is the number of UEs connected to the RN $r, N_{d}$ is the number of UEs connected to the DeNB, $N$ is the total number of UEs in the DeNB cell area, and $N_{R}$ is the number of RNs in the DeNB cell.

Considering a fixed probability for a UE to be attached to an RN $r$, the probability that $n$ out of $N$ UEs active in the DeNB cell is connected to the RN follows the binomial distribution:

$$
P\left(N_{r}=x\right)=\left(\begin{array}{c}
N \\
x
\end{array}\right) * P_{0}^{x} *\left(1-P_{0}\right)^{N-x}
$$

The probability $P_{0}$ corresponds to the portion of the cell area covered by the RNs. For simplicity, it is assumed that the probability is the same for all RNs in the network and equals to $P_{0}=7 \%$ (according to the evaluation assumptions presented in the Appendix).

The mean value $E\left(N_{r}\right)$ and variance $V\left(N_{r}\right)$ for the distribution can be expressed, respectively, as

$$
\begin{aligned}
& E\left(N_{r}\right)=N * P_{0} \\
& V\left(N_{r}\right)=N * P_{0} *\left(1-P_{0}\right)
\end{aligned}
$$

Furthermore, the binomial probability distribution can be approximated with the normal distribution as

$$
P\left(N_{r}=x\right) \cong \frac{1}{\sqrt{2 \pi * V\left(N_{r}\right)}} \exp \left(-\frac{\left(x-E\left(N_{r}\right)\right)^{2}}{2 * V\left(N_{r}\right)}\right)(13)
$$


Based on the properties of the normal distribution, it can be estimated that in $95 \%$ of cases the number of UEs connected to one $\mathrm{RN}$ should be within the range $\left(N_{r}^{\mathrm{MIN}}, N_{r}^{\mathrm{MAX}}\right)$, where

$$
\begin{aligned}
& N_{r}^{\mathrm{Min}}=E\left(N_{r}\right)-2 \sqrt{V\left(N_{r}\right)} \\
& N_{r}^{\mathrm{Max}}=E\left(N_{r}\right)+2 \sqrt{V\left(N_{r}\right)}
\end{aligned}
$$

and the amount of resources assigned to the $\mathrm{RN} r$ backhaul link should be within the range $\left(S_{\mathrm{BH}}{ }^{\mathrm{MIN}}(r)\right.$, $\left.S_{\mathrm{BH}}{ }^{\mathrm{MAX}}(r)\right)$, where

$$
\begin{aligned}
& S_{\mathrm{BH}}^{\mathrm{Min}}(r)=P_{0}-2 \sqrt{\frac{P_{0}\left(1-P_{0}\right)}{N}} \\
& S_{\mathrm{BH}}^{\mathrm{Max}}(r)=P_{0}+2 \sqrt{\frac{P_{0}\left(1-P_{0}\right)}{N}}
\end{aligned}
$$

Finally, the amount of resources utilized by all RNs connected to one DeNB should be within the range $\left(S_{\mathrm{BH}}{ }^{\mathrm{MIN}}, S_{\mathrm{BH}}{ }^{\mathrm{MAX}}\right)$

$$
\begin{aligned}
& S_{\mathrm{BH}}^{\mathrm{Min}}=N_{R} P_{0}-2 \sqrt{\frac{N_{R} P_{0}\left(1-N_{R} P_{0}\right)}{N}} \\
& S_{\mathrm{BH}}^{\mathrm{Max}}=N_{R} P_{0}+2 \sqrt{\frac{N_{R} P_{0}\left(1-N_{R} P_{0}\right)}{N}}
\end{aligned}
$$

where $N_{R} P_{0}$ is the probability that a UE is served by any of the RNs in the network.

The estimation of the most probable minimum and maximum amounts of resources assigned for backhaul links of a single RN and $N_{R}$ RNs is presented in Figure 4. It is worth noting that there is very wide dynamic range of resource assignment to the RN backhaul links. For example, for the case with 25 UEs per DeNB cell area $(N$ $=25$ ), a single RN should have access to $0-17 \%$ of all the DeNB resources. At the same time, all RNs connected to the one DeNB should in total have access to $51-88 \%$ of the resources available at DeNB. Such wide dynamic range of possible resource allocation imposes the need for dynamic sharing of resources between RNs and directly served UEs (i.e., between the backhaul and direct links). The resource sharing secures that resources allocated to RN backhaul links can be reused by the directly served UEs in case only few UEs are connected to the RNs and avoid that these resources are wasted. Furthermore, by comparing the dynamic ranges of resource allocation for a single RN and for all RNs, it becomes obvious that dynamic sharing of resources between RNs (i.e., between backhaul links of different RNs) is also needed to support the different possible user allocations.

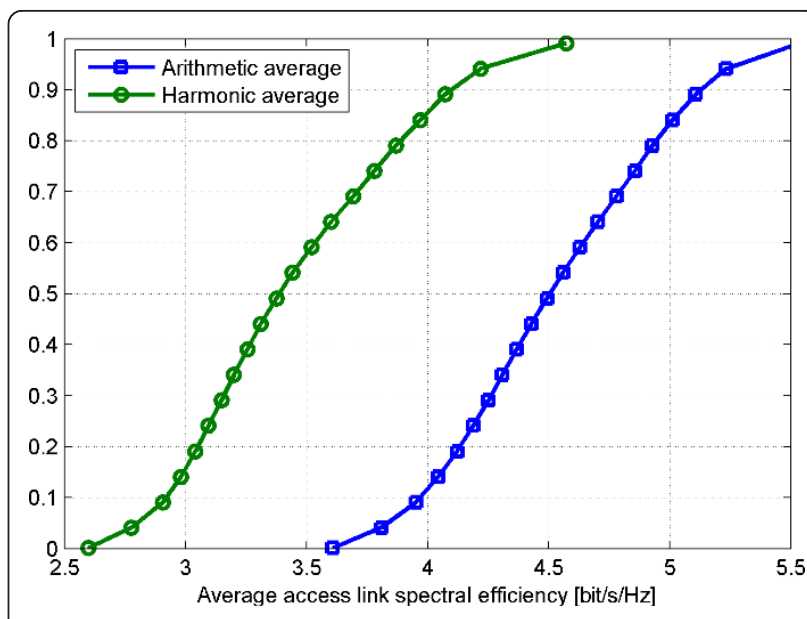

Figure 4 Comparison of arithmetic and harmonic access link capacity estimation (for evaluation assumptions see Appendix).

If dynamic resource sharing between direct and backhaul links and between backhaul links of different RNs is not enabled, the resource assignment may not be fair with respect to all UEs as well as limit the overall system performance. Specifically, without the dynamic resource sharing, if UE allocation to DeNB and RNs is strongly biased towards one of the nodes, the UEs at the overcrowded node experience low resource availability while the UEs at other nodes experience high resource availability. As a result, high performance non-uniformity can be observed.

\subsubsection{Resource partitioning at $R N$}

The second guideline (G2) considers the interconnection of the backhaul and access links via a buffer at the RN. The RN cannot send to the target node more data, than it has received from the source node (not more than the amount of data stored in the buffer). Likewise, the RN cannot receive data from the source node, if its buffer is full. In order to maximize the efficiency of resource utilization the backhaul and access link throughputs should be on average equal

$$
T\left(j_{0}, r\right) * \sigma(r, u)=T(r, u)
$$

where $T\left(j_{0}, r\right)$ is the throughput on the backhaul link of the $\mathrm{RN} r, \sigma(r, u)$ is the fraction of the backhaul link throughput of RN r used by the UE $u$, and $T(r, u)$ is the throughput available for the UE $u$ on the access link to $\mathrm{RN} r$.

Considering that throughput is a product of link SE and resource availability for the link formula (20) can be formulated as

$$
R_{\mathrm{BH}}(r) * \mathrm{SE}\left(j_{0}, r\right) * \sigma(r, u)=R_{\mathrm{AC}}(r) * S(u) * \mathrm{SE}(r, u)
$$


where

$$
R_{\mathrm{BH}}(r)=R * S_{\mathrm{BH}}(r)
$$

where $R_{\mathrm{BH}}(r)$ and $R_{\mathrm{AC}}(r)$ are the amounts of resources assigned, respectively, for operation of the backhaul and access link of RN $r$. Based on (21), the most efficient proportion of resource partitioning for backhaul and access links $\rho(r)$ is defined as

$$
\rho(r)=\frac{R_{\mathrm{AC}}(r)}{R_{\mathrm{BH}}(r)}=\frac{\operatorname{SE}\left(j_{0}, r\right) * \sigma(r, u)}{\operatorname{SE}(r, u) * S(u)}
$$

The fraction of the backhaul link throughput of RN r used by the UE $u$ is then given by

$$
\sigma(r, u)=\frac{R_{\mathrm{AC}}(r) * S(u) * \mathrm{SE}(r, u)}{R_{\mathrm{BH}}(r) * \mathrm{SE}\left(j_{0}, r\right)}
$$

For the maximum efficiency of resource utilization on the backhaul link, the sum of $\sigma(r, u)$ over all UEs connected to RN $r$ should be equal to 1 , thus after simplifications

$$
\sigma(r, u)=\frac{\sigma(r, u)}{\sum_{u \in U(r)} \sigma(r, u)}=\frac{S(u) * \operatorname{SE}(r, u)}{\sum_{u \in U(r)} S(u) * \operatorname{SE}(r, u)}
$$

By combining formulas (23) and (25), there is

$$
\rho(r)=\frac{\operatorname{SE}\left(j_{0}, r\right)}{\operatorname{SE}(r, u) * S(u)} \frac{S(u) * S E(r, u)}{\sum_{u \in U(r)} S(u) * \operatorname{SE}(r, u)}
$$

and after simplification

$$
\rho(r)=\frac{\operatorname{SE}\left(j_{0}, r\right)}{\sum_{u \in U(r)} S(u) * \operatorname{SE}(r, u)}
$$

Finally, assuming the RF resource assignment policy, as defined by Equation (4)

$$
\rho(r)=\frac{\operatorname{SE}\left(j_{0}, r\right)}{\operatorname{Avg}(\operatorname{SE}(r, u))}
$$

where $\operatorname{Avg}(\operatorname{SE}(r, u))$ is the average $\mathrm{SE}$ on the access link calculated over the links of the UEs connected to the RN $r$. If a full buffer traffic model is assumed to be used by the users, the average access link SE is asymptotically equal to the arithmetic average calculated over the whole RN coverage area (29). On the other hand, if a more realistic finite buffer traffic model is assumed, it is required to consider that the UEs with lower SE require more time to transfer a certain amount of data, than the UEs with higher SE (assuming low or no mobility for the users). This implies that, following the analysis presented in [20], the $\operatorname{Avg}(\operatorname{SE}(r, u))$ is asymptotically equal to the harmonic average of the spectral efficiencies in the RN cell (30). Finally, if sufficient mobility is assumed for the users with finite buffer traffic, the impact of SE on transmission time can be neglected and again the arithmetic average can be used

$$
\begin{aligned}
& \rho_{\text {FullBuffer }}(r) \cong \frac{\operatorname{SE}\left(j_{0}, r\right)}{E(\operatorname{SE}(r, u))} \\
& \rho_{\text {FiniteBuffer }}(r) \cong \operatorname{SE}\left(j_{0}, r\right) * E\left(\operatorname{SE}(r, u)^{-1}\right)
\end{aligned}
$$

Comparison of the two averaging options is shown in Figure 5. The use of harmonic averaging results on average in $24 \%$ lower average SE for the RN access link. This is because of higher impact of the users' links with low SE (longer download time). This means that assuming very low or no mobility for users approximately $33 \%$ more resource would be needed for the RN access link in order to achieve balanced backhaul-access link capacities. Assuming that very low or no user mobility is not common in mobile networks further in this article the arithmetic average-based access link capacity estimation is used.

The formula for the optimum RN backhaul-access link resource partitioning $\rho(r)$ depends on the quality (in terms of SE) of the two links. Figure 6 presents distribution of the estimated values for the $\rho(r)$ parameter assuming the following resource allocation schemes:

(a) completely different sets of resources assigned to the neighboring RNs (Reuse- $N_{R}$ between relay cells), hence inter-RN interference does not occur (neither access-to-access link nor access-to-backhaul link);

(b) a common set of resources assigned to all RNs' access links (Reuse-1 between relay cells), hence

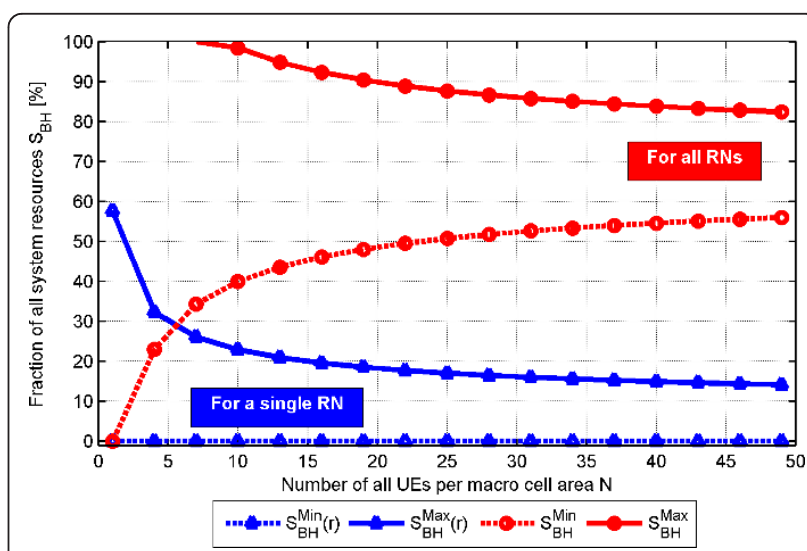

Figure 5 Dynamic ranges of amount of resources assigned to backhaul links of a single RN and all RNs in a cell (for evaluation assumptions see Appendix) 


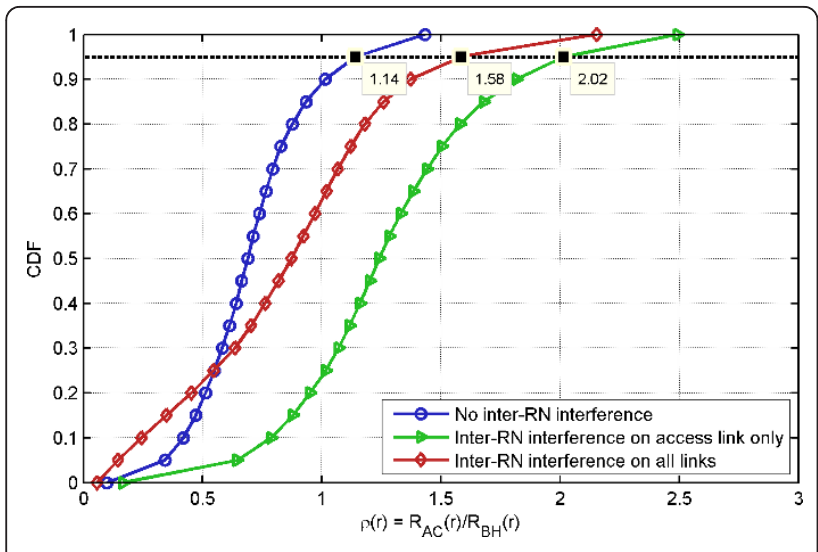

Figure 6 Distribution of the optimal backhaul-access link resource partitioning (for evaluation assumptions see Appendix)

inter-RN interference occurs only between access links (access-to-access link interference only);

(c) resources used for backhaul link of some RNs reused for access links of other RNs, and vice versa, hence inter-RN interference occurs between the access links as well as between access and backhaul links (access-to-access and access-to-backhaul link interference).

Coordination of the inter-RN interference with resource reuse in frequency domain is analyzed in details in Section 4.2.

To practically avoid transmission limitation on the RN access link, the link should be configured with approximately 114-201\% (95 percentile values for $\rho(r)$ as shown in Figure 6) of the amount of resources assigned to the $\mathrm{RN}$ backhaul link $R_{\mathrm{BH}}(r)$ (depending on the resource reuse scheme between RN cells). Considering the earlier estimated number of at most $17 \%$ of system resources to be used for the backhaul link of a single $\mathrm{RN}\left(S_{\mathrm{BH}}{ }^{\text {Max }}\right.$ ( $r$ ) for $N=25 \mathrm{UEs} /$ sector, see Figure 5), this gives approximately $19-34 \%$ of systems resources that should be assigned to access link of an $\mathrm{RN}\left(R_{\mathrm{AC}}(r)\right)$. Yet, if we consider 10 RNs per each DeNB cell $\left(N_{R}=10\right)$, it is clear that Reuse- $N_{R}$ is not possible to be achieved since it would require $N_{R} * 34 \%=340 \%$ of the system resources, thus full elimination of the inter-RN interference is not possible. This means that inter-RN interference needs to be considered at least at some level and that the actual amount of resources that should be assigned to each access link is rather at the high end of the given range.

\subsection{Resource partitioning schemes}

Typically, two backhaul-access link resource partitioning schemes are considered for RNs. Those are resource partitioning in time and frequency domain that correspond to in-band and out-band operations, respectively. In addition, also the so-called hybrid or mixed configuration can be used, as discussed in [40]. Each of the partitioning schemes brings restrictions to resource assignment for RNs, those restrictions are discussed next considering the resource allocation criteria derived in Section 3.2.

\subsubsection{In-band configurations}

In the LTE-Advanced system, the in-band resource partitioning is based on the Multimedia Broadcast over Single Frequency Network (MBSFN) sub-frames [41]. If an RN configures some of its sub-frames as the MBSFN sub-frames, the UEs connected to this RN are informed that during those sub-frames they should not expect any communication to be exchanged on the access link. Since in the MBSFN sub-frames the RN is not required to transmit reference signals, it is free to communicate then with the DeNB on the backhaul link.

The use case of the in-band resource partitioning is the possibility to operate RNs on a single frequency carrier. This is especially important for network operators having just one carrier.

The in-band resource partitioning has, however, also several drawbacks. First of all, communication on both the backhaul and access links is not continuous in time, thus additional forwarding delay is introduced. This may be critical for delay sensitive traffic such as, e.g., voice over IP. Second, with time domain multiplexing protective transmission gaps need to be introduced at the transmission-reception switching instants (Figure 7). Furthermore, because the RN cannot receive the control data from the DeNB while transmitting the control data to its connected UEs (as also shown in Figure 7) a new control channel needs to be implemented. It is estimated that the total overhead of the in-band relaying in the case of 10 RNs per DeNB cell is at the level of $12 \%$ of the wireless backhaul link capacity [40]. The overhead reduces the backhaul link spectral efficiency $\mathrm{SE}(d, r)$, and it can be included into the bandwidth efficiency $\mathrm{BW}_{\text {eff }}$ (Table 1).

Last but not least, utilization of the MBSFN subframes imposes resource allocation restrictions. According to the current specification on the use of MBSFN sub-frame in LTE, only up to six out of ten sub-frames in a radio frame can be configured as the MBSFN subframes. This means that the maximum amount of resources available for backhaul link operation of all RNs in a DeNB cell is limited to $60 \%$. As estimated in the previous section, the $\mathrm{RN}$ backhaul link resource requirement in case of 10 RNs per DeNB cell deployment is in the range of $51-88 \%$. This creates the threat that in case of such dense RN deployments resource availability for the backhaul link would be limited. 


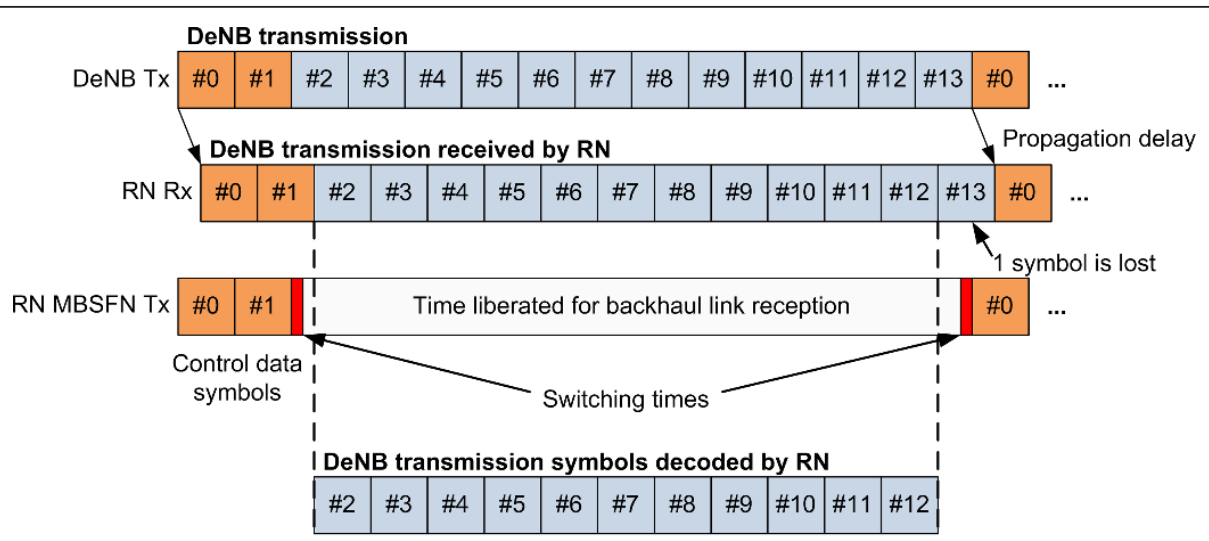

DeNB transmission

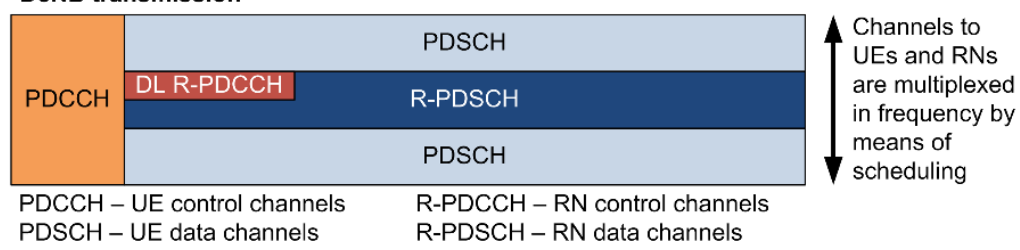

Figure 7 In-band backhaul link operation using MBSFN sub-frames

\subsubsection{Out-band configurations}

With growing system capacity demands, multi-carrier scenarios are getting more and more relevance. To satisfy the high data rate demands of users, network operators are pushed to invest in additional spectrum for their networks. With such trend the RNs are also required to operate on multiple carriers.

The out-band relaying assumes the backhaul-access link resource partitioning to be done in the frequency domain. This means that the backhaul link is operated on a different frequency carrier (or carriers) than the access link. Typically, it is assumed that such configuration provides perfect protection from the loop interference. This is, however, not a valid assumption if the backhaul and access links are operated on adjacent carriers. In such case, power leaking between the carriers needs to be considered, and it is caused by the transmitter and receiver hardware imperfections and characterized by the so-called adjacent channel interference ratio (ACIR) [36]. The ACIR parameter defines what fraction of the power transmitted in one frequency carrier can be measured by a receiver in an adjacent frequency carrier. It is the product of both the transmitter and receiver hardware imperfections (e.g., of their RF filters). For

Table 1 Bandwidth efficiency with in-band backhaul link

\begin{tabular}{lllll}
\hline & \multicolumn{4}{l}{ Number of RNs per DeNB sectors } \\
\cline { 2 - 5 } & $\mathbf{0}$ & $\mathbf{1}$ & $\mathbf{4}$ & $\mathbf{1 0}$ \\
\hline $\mathrm{BW}_{\mathrm{eff}}[\mathrm{b} / \mathrm{s} / \mathrm{Hz}]$ & 0.87 & 0.81 & 0.79 & 0.76 \\
\hline
\end{tabular}

the case of $\mathrm{RN}$ loop interference, the value for ACIR is $42.5 \mathrm{~dB}[40,42,43]$.

The degradation of the backhaul link SINR, caused by the inter-carrier loop interference, as a function of the backhaul-access antenna isolation (AI), is given as

$$
\Delta \operatorname{SINR}=\frac{\operatorname{SINR}\left(j_{0}, r\right)}{\operatorname{SINR}_{0}\left(j_{0}, r\right)}=\left(1+\frac{P_{\mathrm{Tx}}(r) * \operatorname{SINR}_{0}\left(j_{0}, r\right)}{\operatorname{AI} * \operatorname{ACIR} * P_{\mathrm{Rx}}\left(j_{0}, r\right)}\right)^{-1}
$$

where $\operatorname{SINR}_{0}\left(j_{0}, r\right)$ is the SINR experienced on the backhaul link in case of perfect loop interference protection, $\operatorname{SINR}\left(j_{0}, r\right)$ is the actually experienced SINR, and $P_{\mathrm{Tx}}(r)$ is the transmission power of the RN $r$.

Based on the above equation, it can be estimated that the case of a typical RN deployed at the edge of a DeNB cell (as presented in Appendix, $P_{\mathrm{Tx}}(r)=30 \mathrm{dBm}, \mathrm{SINR}_{0}$ $\left.=16 \mathrm{~dB}, P_{\mathrm{Rx}}\left(j_{0}, r\right)=-65 \mathrm{dBm}\right) 60 \mathrm{~dB}$ antenna isolation is needed to achieve the backhaul link SINR degradation not higher than $0.5 \mathrm{~dB}$. At the same time, AI at the level of $50 \mathrm{~dB}$ results in approximately $4 \mathrm{~dB}$ SINR degradation and $40 \mathrm{~dB}$ AI results in $12 \mathrm{~dB}$ SINR degradation [40]. Furthermore, the SINR degradation increases with DeNB-RN distance due to decreasing backhaul link received signal power $P_{\mathrm{Rx}}(d, r)$. Thus, there is the need to either provide sufficient antenna isolation for out-band relays or not allowing allocation of the backhaul and access links on adjacent frequency carriers.

An additional issue of the out-band relaying is that with fixed carrier sizes (as today assumed in LTE) it 
does not allow flexible resource partitioning between the backhaul and access links in the frequency domain. In the LTE system, there are only few carrier sizes specified $(1.4,3,5,10,15$, and $20 \mathrm{MHz})$. A solution to this problem is the carrier aggregation (CA) technique included in the LTE-Advanced system [44-46]. With CA multiple carriers can be used simultaneously for communication on each link, i.e., it can be applied to backhaul, access, and direct links. By enabling the CA technique for RNs proportions of resources assigned to the backhaul and access links can be controlled with higher flexibility.

Considering the deployment of $10 \mathrm{RNs}$ and 25 UEs per DeNB cell area, we have seen in Section 3.1.2 that an RN should be assigned with 19-34\% of system resources for its access link operation. In case of a system with $100 \mathrm{MHz}$ total bandwidth (maximum allowed in LTE-Advanced Release-10) and CA enabled, the RN could be assigned with $20 \mathrm{MHz}$ primary access link carrier and $15 \mathrm{MHz}$ secondary access link carrier. Such configuration would enable dynamic scaling of the RN's access link resources in range of 20-35\%. Alternatively, the RN could be configured with $2 \times 20 \mathrm{MHz}$ carriers for simplicity. At the same time, the remaining $60 \mathrm{MHz}$ of the system bandwidth could be used for allocation of the backhaul link. As estimated in Section 3.1.1, a single RN should utilize up to $17 \%$ of system resources for its backhaul link. Considering this, the backhaul link could be allocated on a single $20 \mathrm{MHz}$ carrier (up to $20 \%$ of system resources) or distributed on multiple carriers. The later approach can provide higher flexibility for resource sharing at the DeNB (i.e., between backhaul and direct links and between backhaul links of different RNs). This aspect is discussed in more details in Section 4.1.

\subsubsection{Hybrid configurations}

The out-band relaying can provide certain gains over the in-band relaying scheme: a throughput gain in case of non-adjacent carrier allocation because of the overhead introduced by the in-band relaying operation and the lower retransmission delay as explained in Section 3.2.1. However, if the number of carriers available in the system is low (e.g., two carriers), the available carrier sizes may not give sufficient flexibility for an efficient resource partitioning in frequency domain. In such case a useful approach may be the hybrid relaying mode [40]. In this configuration, $\mathrm{RN}$ utilizes one or more carriers with the in-band partitioning for both backhaul and access links, and in addition can utilize other carrier(s) for the out-band backhaul and/or access link operation.

The hybrid partitioning scheme combines the advantages of both the in-band and out-band partitioning schemes, at the same time minimizing their disadvantages. Specifically, the flexibility of in-band resource partitioning is available as well as the protection against the loop interference from adjacent carriers while the secondary backhaul carriers enable lower retransmission delay for delay sensitive traffic.

Considering the resource allocation framework presented in Section 3.1, in case of a system with just two carriers, the following configuration is recommended: four to seven -out of ten sub-frames on the in-band carrier configured for the access link operation (i.e., 20-35\% of all system resources) and rest of the resources available for backhaul link operation (i.e., $65-80 \%$ of all system resources). Such configuration should give sufficient flexibility for dynamic resource allocation, to support different distributions of resource requirements.

\subsubsection{Comparison of resource partitioning schemes}

Table 2 summarizes the configurations for in-band, outband, and hybrid resource partitioning discussed above. The in-band resource partitioning scheme provides the lowest maximum amount of resources available for backhaul links. This may be problematic in dense RN deployments. On the other hand, the resolution of the access-backhaul link resource partitioning is good compared to the out-band case. The out-band resource partitioning scheme can provide high maximum resource availability of the backhaul link; however, the resolution of resource partitioning is poor, especially with low number of carriers available. Finally, the hybrid configuration can provide both high resource availability for the backhaul link and good resource partitioning resolution. It is, however, burdened with the aforementioned MBSFN overhead on the in-band carrier(s). Based on the presented analysis it can be recommended to use:

- the in-band configuration in single-carrier systems as then this is the only option available,

- the out-band configuration in multi-carrier systems with high number of carriers available (e.g., 5), when the needed resource partitioning resolution can be satisfied with aggregation of sufficient number of carriers,

\section{Table 2 Comparison of resource partitioning schemes}

\begin{tabular}{llll}
\hline & $\begin{array}{l}\text { Resolution of the } \\
\text { resource } \\
\text { partitioning (\%) }\end{array}$ & $\begin{array}{l}\text { Max. } \\
\text { backhaul } \\
\text { resources } \\
\text { (\%) }\end{array}$ & Other comments \\
\hline In-band & 10 & 60 & MBSFN overhead \\
\hline Out-band $50^{a}$ & $50^{a}$ & $\begin{array}{l}\text { High antenna isolation } \\
\text { required in single-band } \\
\text { operation }\end{array}$ \\
& $20^{\mathrm{b}}$ & $80^{\mathrm{b}}$ & $\begin{array}{l}\text { MBSFN overhead } \\
\text { High antenna isolation } \\
\text { required in single-band } \\
\text { operation }\end{array}$ \\
& $5^{\mathrm{a}}$ & $80^{\mathrm{a}}$ & \\
& $2^{\mathrm{b}}$ & $92^{\mathrm{ab}}$ &
\end{tabular}

an case of 2 carriers available in the system.

${ }^{\mathrm{b}}$ In case of 5 carriers available in the system. 
- the hybrid configuration in multi-carrier systems with low number of carriers available (e.g., 2), when other configurations are not practical.

\section{Resource coordination in multi-carrier systems}

The multi-carrier operation provides two main benefits for relay nodes. First of all, with multiple frequency carriers more resources are available and they can be assigned with higher flexibility as discussed in the previous section. Second, it enables interference coordination solutions in frequency domain that may lead to improved quality of the backhaul and access links. The following sections present how to take advantage out of it.

\subsection{CA and load balancing}

As mentioned in the previous section, in case of multicarrier scenarios resources assigned for backhaul links may be either cumulated on a minimum number of carriers (in the extreme case a single carrier) or distributed over multiple carriers. Considering the average resource requirements for each link both approaches can provide similar results. However, if fluctuations of the resource requirements are considered, e.g., in form of a non-uniform UE distribution or variation in the service demand of the active UEs, the distributed resource allocation allows higher diversity of load per carrier and the possibility to utilize this diversity by inter-carrier load balancing.

To illustrate the carrier load-balancing mechanisms, let us consider a carrier with backhaul links allocated to it. The load on the carrier, defined as the amount of requested resources, can be estimated as

$$
L(c)=\sum_{r \in R(c)} L(r, c)
$$

where $L(c)$ is the total load generated on the carrier $c$ by the set of RNs $R(c)$ having backhaul links allocated to the carrier and $L(r, c)$ is the load generated by each of the RNs.

Assuming equal distribution of backhaul load between all carriers assigned to the $\mathrm{RN} r$ we have that

$$
L(r, c)=\frac{L(r)}{N_{C A}(r)} \Rightarrow L(c)=\sum_{r \in R(c)} \frac{L(r)}{N_{C A}(r)}
$$

where $L(r)$ is the total backhaul load required by the $\mathrm{RN} r$, and $N_{\mathrm{CA}}(r)$ is the number of aggregated carriers for the RN $r$.

Assuming that the load generated by an $\mathrm{RN} L(r)$ is directly related to the amount of resources the $\mathrm{RN}$ should be assigned with according to the criteria specified in Section 3.1, its probability distribution function (PDF) can be approximated with the normal distribution as in Equation (13). If so, the PDF of the cumulated load generated by all the RNs active on the carrier $c$ is also normal distributed with the following expected value and variance:

$$
\begin{aligned}
& E(L(c))=\sum_{r \in R(c)} \frac{E(L(r))}{N_{\mathrm{CA}}(r)} \\
& V(L(c))=\sum_{r \in R(c)} \frac{V(L(r))}{N_{\mathrm{CA}}(r)^{2}}
\end{aligned}
$$

Assuming that the RNs are statistically identical, i.e., probability for a UE to be connected to each of the RNs is the same and each RN aggregates the same number of carriers

$$
\begin{aligned}
& E(L(c))=E(L(r)) \frac{N_{R}(c)}{N_{\mathrm{CA}}} \\
& V(L(c))=V(L(r)) \frac{N_{R}(c)}{N_{\mathrm{CA}}^{2}}
\end{aligned}
$$

where $N_{R}(c)$ is the number of RNs with backhaul links active on the carrier $c$ (cardinality of $R(c)$ ).

Assuming next that all RNs are uniformly distributed on $N_{c}$ carriers, there is

$$
\begin{aligned}
& N_{R}(c)=N_{R} * \frac{N_{\mathrm{CA}}}{N_{C}} \\
& E(L(c))=E(L(r)) * \frac{N_{R}}{N_{\mathrm{CA}}} * \frac{N_{\mathrm{CA}}}{N_{C}}=E(L(r)) * \frac{N_{R}}{N_{C}} \\
& V(L(c))=V(L(r)) * \frac{N_{R}}{N_{\mathrm{CA}}^{2}} * \frac{N_{\mathrm{CA}}}{N_{\mathrm{C}}}=V(L(r)) * \frac{N_{R}}{N_{\mathrm{CA}} * N_{\mathrm{C}}}
\end{aligned}
$$

Based on the above equations, two conclusions can be made. First of all, the number of carriers aggregated per RN does not influence the average load per carrier. Thus, on average performance of RNs with and without CA should be the same. However, if RNs use CA on the backhaul link, the variance of the generated load per carrier can greatly be reduced. As depicted in Figure 8, with higher variance of carrier load the probability of carrier overloading increases. As estimated for the scenario with 10 RNs and 25 UEs per DeNB cell, if CA is not used on the backhaul link, there is 19\% probability of overloading a single carrier. If CA is enabled, the overloading probability can be reduced to $11 \%$ with two carriers aggregated per RN and even down to $2 \%$ with five carriers aggregated. At the same time the average carrier load is at the level of $70 \%$ irrespectively of the CA. 

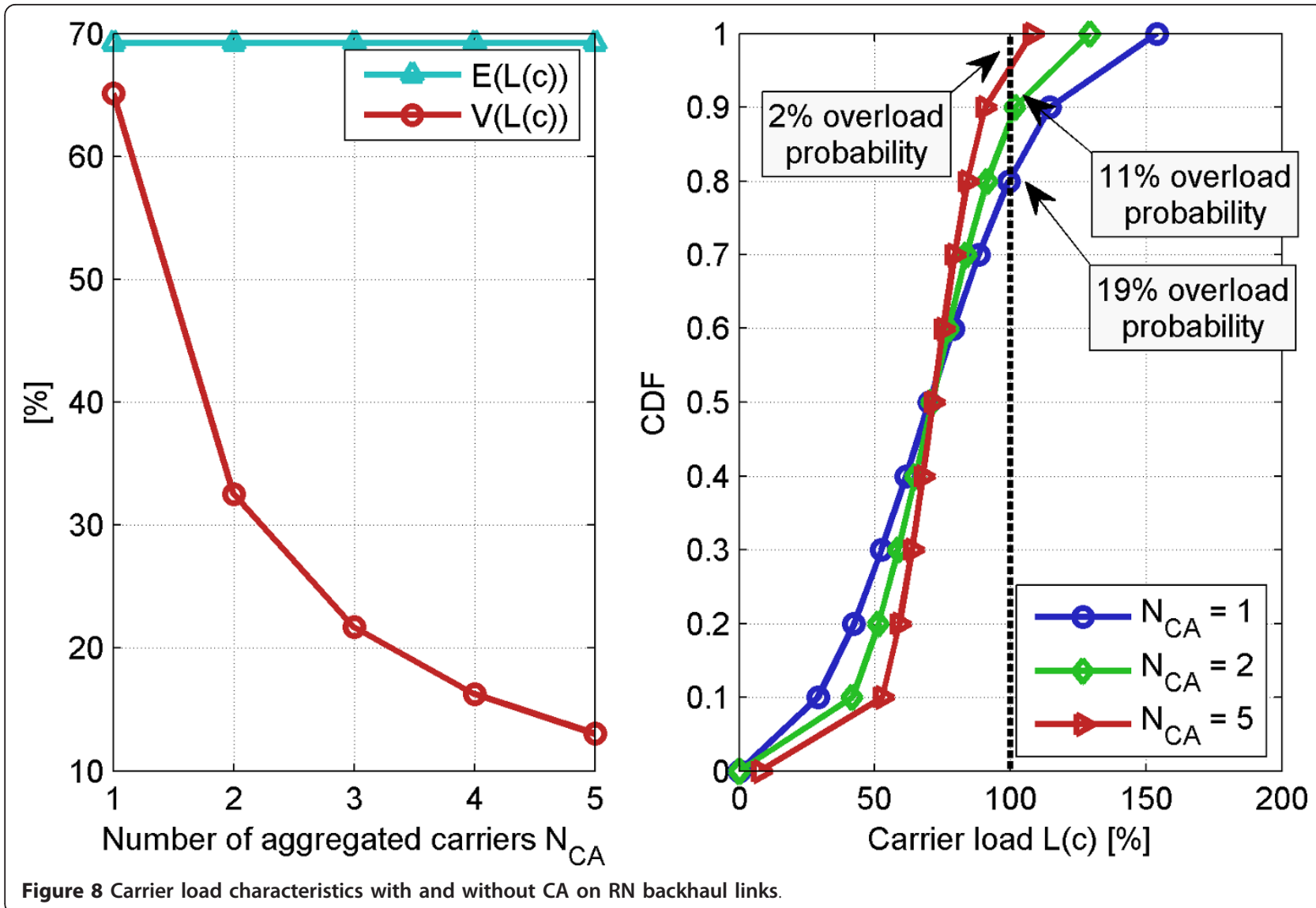

Figure 8 Carrier load characteristics with and without CA on RN backhaul links

4.2. Interference coordination schemes

RNs are classified as low power nodes, with similar transmission characteristics as femto or pico cells. Their transmission power $(1 \mathrm{~W} / 10 \mathrm{MHz})$ is significantly lower than in case of macro stations $(40 \mathrm{~W} / 10 \mathrm{MHz})$, thus their serving areas are also smaller. The range of a relay cell is small; however, if RNs are densely deployed (e.g., to provide continuous coverage extension), the inter-cell interference might be significant. The inter-cell interference between RNs can manifest itself in two ways in downlink (Figure 9): as the access-to-access (A2A) link interference or as the access-to-backhaul (A2B) link

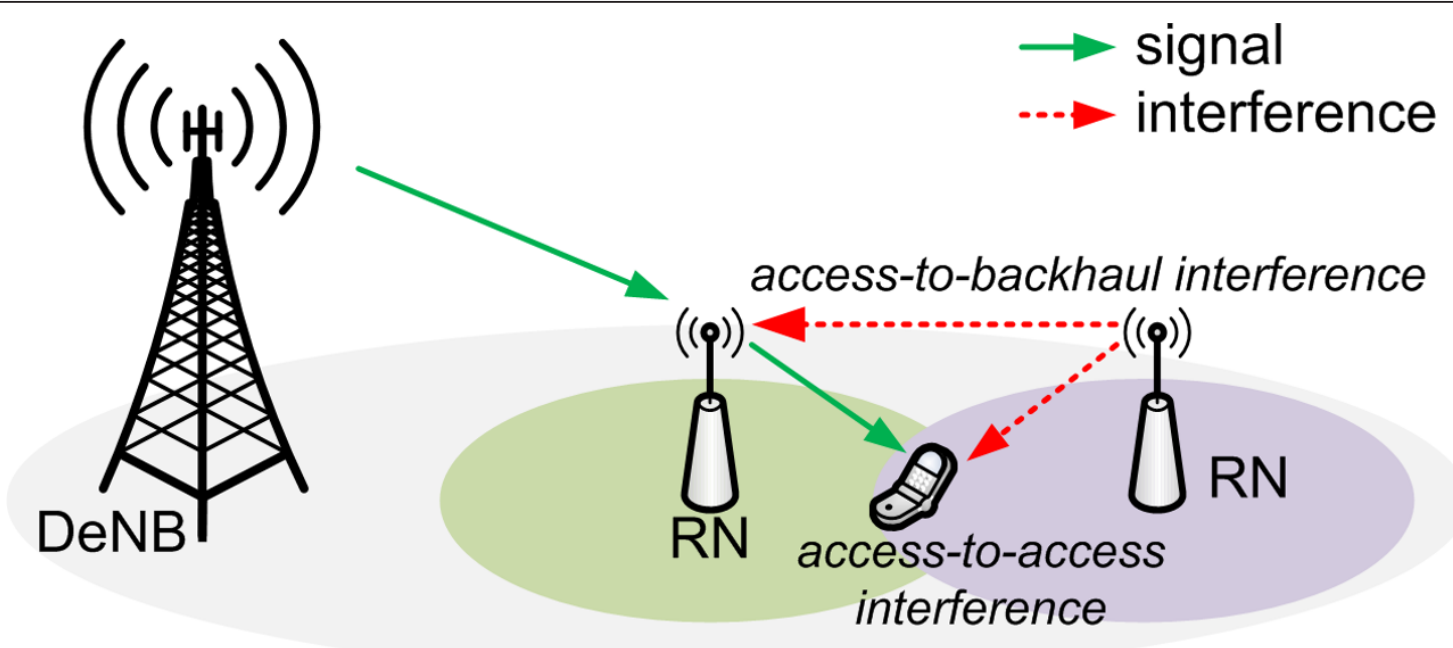

Figure 9 Interference coupling in downlink. 
interference. The A2A interference occurs on the radio resources used on the access links at neighboring RNs while the A2B interference on radio resources used on the access link at the aggressor RN and on the backhaul link at the victim RN. Both interference effects negatively influence the RN performance and thus need to be mitigated.

Several inter-cell interference coordination (ICIC) schemes have already been proposed for different types of cellular systems (traditional macro-only, heterogeneous, relay-enhanced). The designed solutions start with fixed soft and fractional frequency reuse schemes $[47,48]$ and ranges through different kinds of dynamic distributed and autonomous coordination schemes $[49,50]$. The common characteristic of all those solutions is the minimization of interference perceived by UEs and hence focuses on controlling the A2A type of interference. In case of relay-enhanced networks, however, the A2B interference can become critical. Considering this, the ICIC solutions for RNs should not only focus on minimizing the interference at UEs, but also the interference at RNs.

The issue of interference on the RN backhaul link was noticed in [51], and in [52] a grouping based solution for minimizing interference between single-carrier operated in-band RNs was proposed. In [53], ICIC solutions for multi-carrier operated out-band RNs based on coordinated hard frequency reuse carrier allocation are proposed. The ICIC approach proposed in [53] is centralized, i.e., it assumes that the DeNB fully controls carrier allocation for backhaul and access links of all connected RNs. In [16], similar solutions are also proposed, however, assuming distributed and autonomous control, i.e., shifting of some of the decisions to RNs. In this article, further enhancements and evaluations of the centralized solution are presented.

The issue with ICIC for RNs is that procedures leading to improving backhaul link quality and the procedures leading to improving access link quality are often contradictory. As disjunctive sets of resources are used by backhaul and access links at each $\mathrm{RN}$, the only observed interference is the A2A while the A2B in not present. The reduction of the A2A interference by reallocating the access link of the aggressor $\mathrm{RN}$ to different time/frequency resources may often lead to the creation of new A2B interference (Figure 10). Vice versa, reduction of $A 2 B$ interference may lead to generation of new A2A interference.
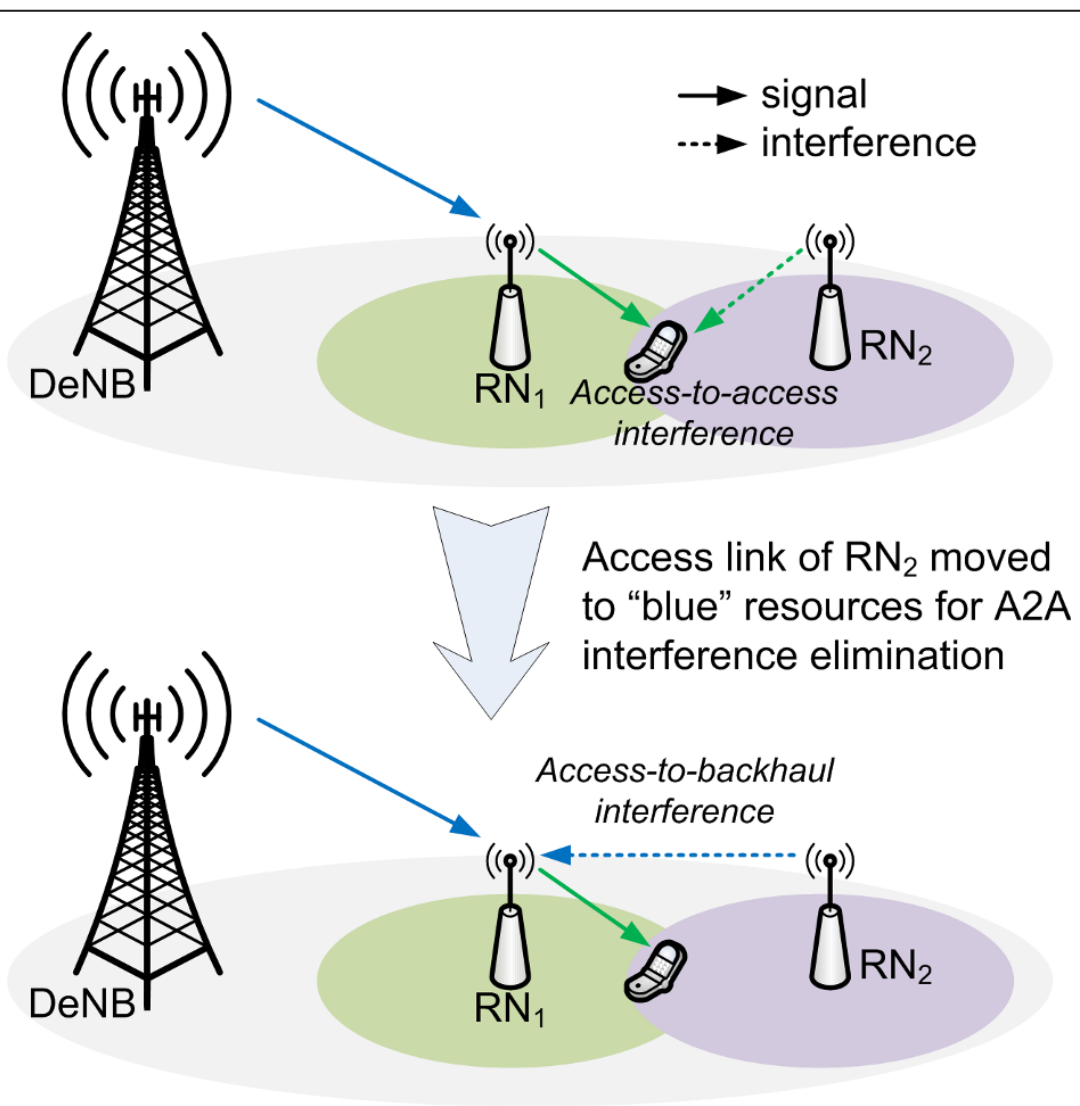

Figure $10 \mathrm{RN}$ interference mitigation dilemma. 
Considering Equation (21), in order to maximize performance of relayed UEs the qualities of both the backhaul and access links need to be improved. To achieve this, the ICIC steps leading to the reduction of the A2A and $\mathrm{A} 2 \mathrm{~B}$ interference need to be balanced.

An ICIC procedure for relay-enhanced systems considering balancing of the A2A/A2B interference mitigation steps is presented in [53]. The procedure proposed there is based on measurements collected by each RN, including signal quality on backhaul and access links and strength of measured interference from neighboring RNs. Based on those measurements, each RN is able to determine, if its performance is endangered and which neighbor RNs are the aggressors. This is done by calculating interference-to-signal ratio (ISR) for each neighbor node on each available carrier, and separately for
RN backhaul and access link (step 1 in Figure 11). The measured ISR is next compared with predefined (e.g., operator specific) threshold levels $\varepsilon_{\mathrm{A} 2 \mathrm{~A}}$ and $\varepsilon_{\mathrm{A} 2 \mathrm{~B}}$, respectively, for A2A and A2B types of interferences (step 2 in Figure 11).

Based on the detected conflicts interference mitigation measures based on coordinated reallocation of resources can be taken (step 3 in Figure 11). There are several options for reconfiguring the RNs suffering from A2A and/or A2B interference, e.g., centralized grouping of the RNs with the strongest interference coupling can be done as proposed in [52], or distributed game-theorybased reconfiguration negations can be done between the affected RNs. For the evaluations presented here, it is assumed that a centralized iterative reconfiguration procedure is used. A centralized unit (DeNB) (1) sorts

Step 1: Collect measurements

(a) Done by RN:

- DeNB signal RSRP on RN backhaul link

- RSRP of A2B interference on all carriers

(b) collected by UEs:

- serving RN signal RSRP

- RSRP of A2A interference on all carriers

Step 2: Detect conflicts

(a) Calculate A2A/A2B ISR for interferers on all carriers

(b) Compare A2A/A2B ISR with $\varepsilon_{\mathrm{A} 2 \mathrm{~A}} / \varepsilon_{\mathrm{A} 2 \mathrm{~B}}$ (ISR $>\varepsilon$ indicates strong interference coupling)

Step 3: Resolve conflicts

Change allocation of carriers to RN backhaul/access links to avoid strong interference (e.g. iteratively)

Figure 11 Multi-carrier ICIC algorithm for out-band relay nodes. 
RNs according to decreasing experienced ISR and (2) sequentially reconfigures them to minimize the cumulated system ISR. For each RN backhaul and/or access link, the carriers are selected, which are burdened with the lowest interference level or the lowest number of conflicts with the neighboring RNs. Reconfigurations of RNs are done in turns up to the point of achieving stable radio conditions in the system.

The proposed ICIC procedure considers separate conditions defining inter-RN conflicts due to the A2A and $A 2 B$ interferences. The conditions are represented by the threshold parameters: $\varepsilon_{\mathrm{A} 2 \mathrm{~A}}$ and $\varepsilon_{\mathrm{A} 2 \mathrm{~B}}$, respectively. The parameters correspond to inversed values of assumed minimum SINR for the access and backhaul links of an RN. By setting proper values for these thresholds it is possible to balance sensitivities of the A2A and A2B interference mitigations, separately.

To determine the $\varepsilon_{\mathrm{A} 2 \mathrm{~A}}$ and $\varepsilon_{\mathrm{A} 2 \mathrm{~B}}$ values that result in the highest performance, different combinations of the thresholds have been tested. It has been determined that the best results are achieved when the A2B interference mitigation threshold $\varepsilon_{\mathrm{A} 2 \mathrm{~B}}$ is higher than the threshold for the $\mathrm{A} 2 \mathrm{~A}$ interference mitigation $\varepsilon_{\mathrm{A} 2 \mathrm{~A}}$ by approximately $16 \mathrm{~dB}$ (Figure 12). This confirms that in case of relay-enhanced systems securing high quality of the wireless backhaul link is more crucial than improvement of the access link quality. However, the reduction of the
A2A interference should not be fully omitted as this may also lead to lower performance. It is assumed that as a baseline the $\varepsilon_{\mathrm{A} 2 \mathrm{~A}}$ and $\varepsilon_{\mathrm{A} 2 \mathrm{~B}}$ should be fixed and determined by the operator at the network planning stage. As a further enhancement, the parameters could be dynamically optimized by the network itself according to the self-optimizing network principle.

\section{Conclusions}

In this article, the problem of resource management in relay-enhanced systems has been discussed. Because of many degrees of freedom, the selection of an optimal resource management scheme is not trivial. In the process, many factors and contradicting mechanisms need to be considered. The analysis presented in this article gives a set of recommendations for the resource assignment in case of a relay-enhanced system.

In a system with relay nodes, three types of links need to be considered and managed in a balanced way. Based on the analysis conducted in this article, it can be concluded that the best performance can be achieved only, if the resource allocation is done in a dynamic way and with high flexibility to reallocate the resources on demand depending on the traffic characteristics.

Three relay node modes of operation (resource partitioning schemes) have been considered: in-band, outband, and hybrid. From the three schemes, the in-band

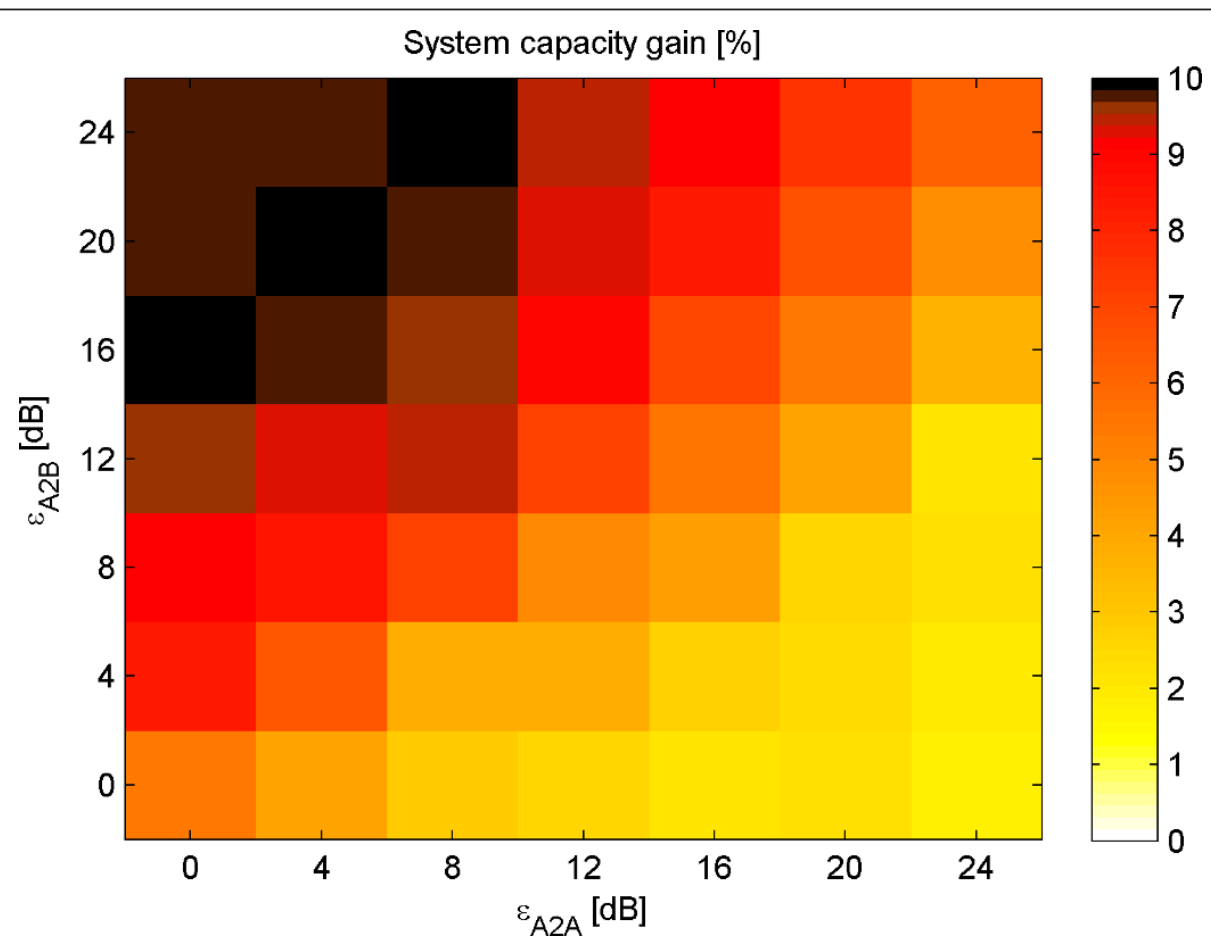

Figure 12 System capacity improvement in relation to thresholds triggering the interference mitigation procedure (for evaluation assumptions see Appendix). 
configuration introduces additional delay, and is burdened with time domain multiplexing overhead and restrictions in resource assignment. It is, however, the only solution possible in case of single-carrier operation. Out-band configuration overcomes weak points of the in-band configuration, but it is most useful in case of systems with high number of carriers and with CA technique implemented. Finally, the hybrid configuration brings together advantages of the in-band and out-band configurations. It is recommended for multi-carrier systems with low number of carriers available (e.g., only two carriers).

Two advanced solutions for resource coordination have been presented in this article. The first one is inter-relay interference coordination. This procedure can improve quality of radio links; however, to achieve good performance a balanced approach to improve backhaul and access link qualities is needed. Finally, also the carrier load-balancing mechanism is presented. The solution is based on CA technique applied to backhaul links. By allowing RNs to operate on multiple frequency carriers, the threat of overloading a single carrier can efficiently be minimized.

At the end, it should be noted that the analysis presented in this article has been done for the simplified case of a network with uniform user distribution and best effort, full buffer traffic model. In the context of a real network, the key findings of the article should be valid in long term, i.e., assuming averaging of the traffic load conditions due to user mobility and variation in time.

\section{Appendix}

The evaluation assumptions used in this article are in line with the widely used methodology defined by the 3GPP and described in [17]. Details of the models are as follows.

\section{Deployment model}

The evaluated network is built on 19 tree-sectored base stations (DeNBs). The base stations are deployed on a regular, hexagonal grid with 1732-m inter site distance between the base stations. In each of the DeNB sectors (cells), there are ten RNs deployed in two tiers as depicted in Figure 13.

Users are deployed uniformly in the system and are indoor, since relays are outdoors a $20-\mathrm{dB}$ penetration loss is assumed on the direct and access links but not on the backhaul link.

\section{Channel model}

The signal power from a transmitting (Tx) node $j$ at a receiving $(\mathrm{Rx})$ node $u$ is modeled as

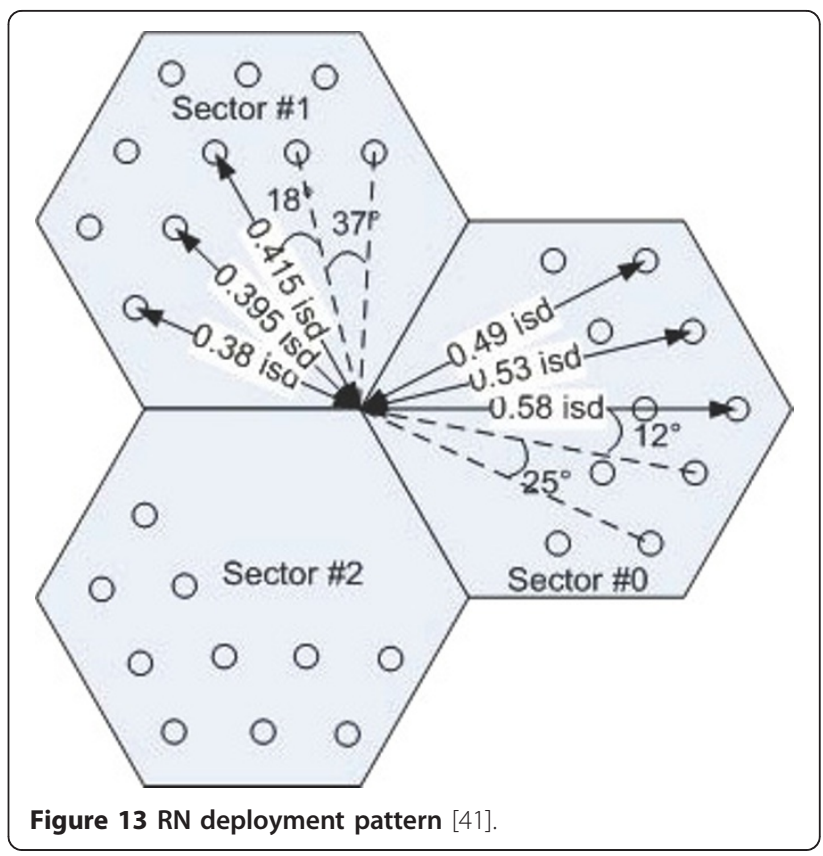

$$
P_{\mathrm{Rx}}(j, u)=P_{\mathrm{Tx}}(j) * \frac{\operatorname{AP}\left(\phi_{\mathrm{Tx}}(j, u)\right) * \operatorname{AP}\left(\phi_{\mathrm{Rx}}(j, u)\right)}{\operatorname{PL}(d(j, u))} * 10^{\psi}
$$

where $\operatorname{AP}\left(\phi_{\mathrm{Tx}}\right)$ and $\operatorname{AP}\left(\phi_{\mathrm{Tx}}\right)$ are the $\mathrm{Tx}$ and $\operatorname{Rx}$ antenna pattern gains, respectively, $\phi_{\mathrm{Tx}}\left[{ }^{\circ}\right]$ is the angle under which the Rx node is visible from the Tx node with respect to the Tx antenna direction, $\phi_{\mathrm{Rx}}\left[^{\circ}\right]$ is the angle under which the Tx node is visible from the $\mathrm{Rx}$ node with respect to the $\mathrm{Rx}$ antenna direction, $\mathrm{PL}(d)$ is the link pathloss as a function of the Rx-Tx node distance $d[\mathrm{~m}]$, and $\psi$ is a random shadow fading.

\section{The antenna pattern and pathloss are modeled as}

$$
\begin{aligned}
& \operatorname{AP}\left(\phi_{\mathrm{Tx}}\right)=\max \left(\alpha_{\mathrm{Tx}} * 10^{\left.-\beta_{T x}\left(\frac{\phi_{\mathrm{Tx}}}{\Phi_{\mathrm{Tx}}}\right)^{2}, \mathrm{AP}_{\mathrm{Min}}\right)}\right. \\
& \operatorname{PL}(d)=\gamma * d^{\delta}
\end{aligned}
$$

where $\alpha, \beta, \gamma, \delta$ are parameters of the channel, $\Phi\left[^{\circ}\right]$ is the angle $3-\mathrm{dB}$ signal attenuation of the antenna pattern, and $\mathrm{AP}_{\mathrm{MIN}}$ is the minimum antenna gain (in backwards direction).

The 3GPP methodology defines models for the direct, backhaul, and access links (see Table 3). For each of the link types, there are LOS and non-line-of-sight (NLOS) components specified. Selection of the LOS/NLOS model is done randomly with respect to a distancedependent probability function $P_{\mathrm{LOS}}(d)$. Values for the parameters describing link models are summarized in 
Table 3 Evaluation assumptions [17]

\begin{tabular}{llll}
\hline Parameter & Direct link & Backhaul link & Access link \\
\hline$\alpha \mathrm{Tx}$ & 25.12 & 25.12 & 3.16 \\
$\alpha \mathrm{Rx}$ & 1.00 & 3.16 & 1.00 \\
$\beta \mathrm{Tx}$ & 1.20 & 1.20 & 0.00 \\
$\beta \mathrm{Rx}$ & 0.00 & 1.20 & 0.00 \\
$\Phi \mathrm{Tx}$ & $70^{\circ}$ & $70^{\circ}$ & $0^{\circ}$ \\
$\Phi \mathrm{Rx}$ & $0^{\circ}$ & $70^{\circ}$ & $0^{\circ}$ \\
$\mathrm{AP}_{\text {Min }} \mathrm{Tx}$ & 0.08 & 0.08 & 1.00 \\
$\mathrm{AP}_{\text {Min }} \mathrm{Rx}$ & 1.00 & 0.03 & 1.00 \\
$\gamma \operatorname{LOS}$ & $1.20 \mathrm{e}+5$ & $1.05 \mathrm{e}+3$ & $12.88 \mathrm{e}+5$ \\
$\gamma$ NLOS & 186.21 & 42.66 & $1.85 \mathrm{e}+5$ \\
$\delta$ LOS & 2.42 & 2.35 & 2.09 \\
$\delta$ NLOS & 4.28 & 3.63 & 3.75 \\
$\psi$ & $0.8^{*}$ RandN( ) & $0.6^{*}$ Rand N() & $1.3^{*}$ RandN() \\
\hline
\end{tabular}

Rand $N($ ), random value with normal probability distribution.

Table 3. Transmission powers of DeNBs and RNs are 40 $\mathrm{W} / 10 \mathrm{MHz}$ and $1 \mathrm{~W} / 10 \mathrm{MHz}$, respectively.

The probability of a LOS link is defined for the direct (DI), backhaul (BH), and access (AC) links, respectively, as [17]

$$
\begin{aligned}
& P_{\mathrm{LOS}}^{\mathrm{DI}}(d)=\min \left(\frac{18}{d}, 1\right) *\left(1-\exp \left(-\frac{d}{63}\right)\right)+\exp \left(-\frac{d}{63}\right) \\
& P_{\mathrm{LOS}}^{\mathrm{BH}}(d)=\min \left(\frac{18}{d}, 1\right) *\left(1-\exp \left(-\frac{d}{72}\right)\right)+\exp \left(-\frac{d}{72}\right) \\
& P_{\mathrm{LOS}}^{\mathrm{AC}}(d)=\frac{1}{2}-\min \left(5 e^{-\frac{156}{d}}, \frac{1}{2}\right)+\min \left(5 e^{-\frac{d}{30}}, \frac{1}{2}\right)
\end{aligned}
$$

SINR-to-SE mapping is based on adjusted Shannon functions specified in [31]. The $2 \times 2$ MIMO (multiple inputs-multiple outputs) operation is assumed for all links, with dynamic switching between beamforming and dual-stream transmission schemes. Based on [31], the mapping function being combination of the mapping functions for the two considered MIMO schemes is

$$
\operatorname{SE}\left(j_{0}, u\right)=\left\{\begin{array}{l}
0: \text { if } \operatorname{SINR}<0.1 \\
\operatorname{Min}\left(8,0.87 * \log _{2}\left(1+\frac{\operatorname{SINR}\left(j_{0}, u\right)}{1.08}\right)\right)
\end{array}\right.
$$

where $\mathrm{SE}$ is given in $\mathrm{bit} / \mathrm{s} / \mathrm{Hz}$ and SINR in linear scale.

\section{Acknowledgements}

The research leading to these results has received founding from the European Commission's 7th Framework Program FP7-ICT-2009 under the grant agreement no. 247223 also referred to as ARTIST4G.

\section{Author details}

${ }^{1}$ Nokia Siemens Networks - Research - Radio Systems, ul. Strzegomska 2-4, 53-611 Wroclaw, Poland 2Department of Wireless Communications, Poznan
University of Technology, Poznan, Poland ${ }^{3}$ Nokia Siemens Networks Research - Radio Systems, St. Martin Strasse 76, 81541 Munich, Germany

\section{Competing interests}

Authors of the article are employees of the Nokia Siemens Networks compaty that is a major telecommunications vendor, and as such is involved in development of concepts for next generation mobile telecommunications systems. The Nokia Siemens Networks company has partially financed the research described in this article and has covered the article processing charge. The research described in this article is also part of the ARTIST4G project co-founded by the European Commision as part of the $7^{\text {th }}$ Framework Program.

Received: 15 September 2011 Accepted: 29 March 2012 Published: 29 March 2012

\section{References}

1. E van der Meulen, Three-terminal communication channel. Adv Appl Probab. 3, 120-154 (1971). doi:10.2307/1426331

2. E van der Meulen, A survey of multi-way channels in information theory: 1961-1976. IEEE Trans Inf Theory. 23(1), 1-37 (1977). doi:10.1109/ TIT.1977.1055652

3. T Cover, AE Gamal, Capacity theorems for the relay channel. IEEE Trans Inf Theory. 25(5), 572-584 (1979). doi:10.1109/TIT.1979.1056084

4. M Sidi, A Segall, A three-node packet radio network. IEEE Trans Commun. 32(12), 1336-1339 (1984). doi:10.1109/TCOM.1984.1096007

5. A Imran, R Tafazolli, Evaluation and comparison of capacities and costs of multihop cellular networks. in Proceedings of the IEEE International Conference on Telecommunications, Marrakech, Maroco, 25-27 May 2009 160-165

6. E Lang, S Redana, B Raaf, Business impact of relay deployment for coverage extension in 3GPP LTE-Advanced. in Proceeding of the IEEE International Conference on Communications Workshops, Dresden, Germany, 14-18 June 2009 1-5

7. P Moberg, P Skillermark, N Johansson, A Furuskaer, Performance and cost evaluation of fixed relay nodes in future wide area networks. in Proceedings of the IEEE 18th International Symposium on Personal, Indoor and Mobile Radio Communications, Athens, Greece, 3-7 September 2007 1-5

8. DC Schultz, B Walke, Fixed relays for cost efficient $4 \mathrm{G}$ network deployments: an evaluation. in Proceedings of the IEEE 18th International Symposium on Personal, Indoor and Mobile Radio Communications, Athens, Greece, 3-7 September 2007 1-5

9. A Bou Saleh, S Redana, B Raaf, J Hämäläinen, On the coverage extension and capacity enhancement of inband relay deployments in LTE-Advanced networks. J Electric Comput Eng. 2010, 1-12 (2010)

10. 3GPP, UTRA-UTRAN Long Term Evolution (LTE) and 3GPP System Architecture Evolution (SAE), Technical paper (2008)

11. IEEE, IEEE Standard for Local and metropolitan area networks; Part 16: Air Interface for Broadband Wireless Access Systems (2009)

12. IEEE, IEEE Standard for Local and metropolitan area networks; Part 16: Air Interface for Broadband Wireless Access Systems; Amendment 1: Multihop Relay Specification (2009)

13. K Wesolowski, Application of MIMO and network coding in two-way relaying applied in LTE. in Proceedings of the IEEE 21st International Symposium on Personal, Indoor and Mobile Radio Communications, Istanbul, Turkey, 26-29 September 2010619-624

14. Y Jing, B Hassibi, Distributed space-time coding in wireless relay networks. IEEE Trans Wirel Commun. 5(12), 3524-3536 (2006)

15. S Valentin, Cooperative relaying and its application-from analysis to prototypes, PhD thesis, (University of Paderborn, 2009)

16. ARTIST4G, D3.2: Advanced relay technical proposals (2010)

17. 3GPP, TR 36.814 V9.0.0: Evolved Universal Terrestrial Radio Access (E-UTRA); further advancements for E-UTRA physical layer aspects (Release 9) (2010)

18. 3GPP, TR 36.806 V9.0.0: Evolved Universal Terrestrial Radio Access (E-UTRA); relay architectures for E-UTRA (LTE-Advanced) (Release 9) (2010)

19. A Bou Saleh, S Redana, B Raaf, T Riihonen, J Hamalainen, R Wichman, Performance of amplify-and-forward and decode-and-forward relays in LTEAdvanced. in Proceedings of the IEEE 70th Vehicular Technology Conference, Anchorage, USA 20-23 September 2009 1-5

20. L Rong, SE Elayoubi, OB Haddada, Impact of relays on LTE-Advanced performance. in Proceedings of the IEEE International Conference on Communications, Cape Town, South Africa, 23-27 May 2010 1-6 
21. K Zheng, B Fan, Z Ma, G Liu, X Shen, W Wang, Multihop cellular networks toward LTE-Advanced. IEEE Veh Technol Mag. 4(3), 40-47 (2009)

22. M Qingyu, A Osseiran, Performance comparison between DF relay and RF repeaters in the cellular system. in Proceedings of the IEEE 67th Vehicular Technology Conference, Singapore, 11-14 May 2008 2021-2025

23. A Hazmi, J Rinne, M Renfors, Cancellation of loop interference with exponential profile using autocorrelation method in OFDM based systems in Proceedings of the IEEE International Conference on Communications Systems, Singapore, 6-9 September 2004 140-144

24. H Hamazumi, K Imamura, N lai, K Shibuya, M Sasaki, A study of a loop interference canceller for the relay stations in an SFN for digital terrestrial broadcasting. in Proceedings of the IEEE Global Telecommunications Conference, San Francisco, USA, 27 November-1 December 2000 167-171

25. A Bou Saleh, Ö Bulakci, Z Ren, S Redana, B Raaf, J Hämäläinen, Resource sharing in relay-enhanced $4 \mathrm{G}$ networks: downlink performance evaluation. in Proceedings of the European Wireless Conference, Viena, Austria, 27-29 April $20111-8$

26. Ö Bulakci, A Bou Saleh, S Redana, B Raaf, J Hämäläinen, Two-step resource sharing and uplink power control optimization in LTE-Advanced relay networks. in Proceedings of the International Workshop on Multi-Carrier Systems and Solutions, Herrsching, Germany, 3-4 May 2011 1-6

27. Ö Bulakci, A Bou Saleh, S Redana, B Raaf, J Hämäläinen, Flexible backhaul resource sharing and uplink power control optimization in LTE-Advanced relay networks. in Proceedings of the IEEE 73rd Vehicular Technology Conference, Budapest, Hungary, 15-18 May 2011 1-6

28. A Bou Saleh, Ö Bulakci, S Redana, B Raaf, J Hämäläinen, Addressing radio resource management challenges in LTE-Advanced relay networks: downlink study. in Proceedings of the VDE 16. ITG Workshop on Mobile Communications, Osnabrück, Germany, 18-19 May 2011 1-6

29. Ö Bulakci, A Bou Saleh, S Redana, B Raaf, J Hämäläinen, Uplink radio resource management challenges in LTE-Advanced relay networks. in Proceedings of the VDE 16. ITG Workshop on Mobile Communications, Osnabrück, Germany, 18-19 May 2011 1-6

30. M Kiiski, LTE-Advanced: the mainstream in mobile broadband evolution. in Proceedings of the European Wireless Conference, Locca, Italy, 12-15 April 2010 983-988

31. PE Mogensen, T Koivisto, KI Pedersen, IZ Kovacs, B Raaf, K Pajukoski, MJ Rinne, LTE-Advanced: the path towards gigabit/s in wireless mobile communications. in Proceedings of the International Conference on Wireless Communication, Vehicular Technology, Information Theory and Aerospace \& Electronic Systems Technology, Aalborg, Denmark, 17-20 May 2009 147-151

32. V Stencel, A Muller, P Frank, LTE-Advanced-a further evolutionary step for next generation mobile networks. in Proceedings of the International Conference Radioelektronika, Brno, Czech Republic, 19-21 May 2010 1-5

33. S Parkvall, E Dahlman, A Furuskar, Y Jading, M Olsson, S Wanstedt, K Zangi, LTE-Advanced-evolving LTE towards IMT-Advanced. in Proceedings of the IEEE 68th Vehicular Technology Conference, Calgary, Canada, 21-24 September 2008 1-5

34. 3GPP, TS 36.300 V10.2.0: Evolved Universal Terrestrial Radio Access (E-UTRA) and Evolved Universal Terrestrial Radio Access Network (E-UTRAN); Overall description; Stage 2 (Release 10) (2010)

35. 3GPP, TS 36.331 V10.1.0: Evolved Universal Terrestrial Radio Access (E-UTRA); Radio Resource Control (RRC); Protocol specification (Release 10) (2011)

36. H Holma, A Toskala, LTE for UMTS-OFDMA and SC-FDMA-Based Radio Access, (John Wiley \& Sons Ltd., Chichester, Unided Kingdom, 2009)

37. P Mogensen, N Wei, IZ Kovacs, F Frederiksen, A Pokhaiyal, KI Pedersen, T Kolding, K Hugl, M Kuusela, LTE capacity compared to the shannon bound. in Poceedings of the IEEE 65th Vehicular Technology Conference, Dublin, Ireland, 22-25 April 2007 1234-1238

38. RK Jain, The Art of Computer Systems Performance Analysis: Techniques for Experimental Design, Measurement, Simulation and Modeling, (Willey, Inc., New York, 1991)

39. Ö Bulakci, S Redana, B Raaf, J Hamalainen, Performance enhancement in LTE-Advanced relay networks via relay site planning. in Proceedings of the IEEE 71st Vehicular Technology Conference, Taipei, Taiwan, 16-19 May 2010 $1-5$

40. J Góra, S Redana, In-band and out-band relaying configurations for dualcarrier LTE-Advanced system. in Proceedings of the IEEE 22nd International Symposium on Personal, Indoor and Mobile Radio Communications, Toronto, Canada, 11-14 September 2011 1820-1824
41. Texas Instruments, R1-091294: On the design of relay node for LTE-Advanced, 3GPP RAN WG1 (2009)

42. 3GPP, TR 36.101 V10.1.1: Evolved Universal Terrestrial Radio Access (E-UTRA); User Equipment (UE) radio transmission and reception (Release 10) (2011)

43. 3GPP, TS 36.104 V10.1.0: Evolved Universal Terrestrial Radio Access (E-UTRA); Base Station (BS) radio transmission and reception (Release 10) (2010)

44. R Ratasuk, D Tolli, A Ghosh, Carrier aggregation in LTE-Advanced. in Proceedings of the IEEE 71st Vehicular Technology Conference, Taipei, Taiwan, 16-19 May 2010 1-5

45. G Yuan, X Zhang, W Wang, Y Yang, Carrier aggregation for LTE-Advanced mobile communication systems. IEEE Commun Mag. 48(2), 88-93 (2010)

46. M Iwamura, K Etemad, MH Fong, R Nory, R Love, Carrier aggregation framework in 3GPP LTE-Advanced. IEEE Commun Mag. 48(8), 60-67 (2010)

47. J Wang, Inter-cell interference coordination based on soft frequency reuse for relay enhanced cellular network. in Proceedings of the IEEE 21st International Symposium on Personal, Indoor and Mobile Radio Communications, Istanbul, Turkey, 26-29 September 2010 2304-2308

48. G Boudreau, J Panicker, S Vrzic, Interference coordination and cancellation for 4G networks. IEEE Commun Mag. 47(4), 74-81 (2009)

49. J Ellenbeck, C Hartmann, L Berlemann, Decentralized inter-cell interference coordination by autonomous spectral reuse decisions. in Proceedings of the European Wireless Conference, Prague, Czech Republic, $22-25$ June 2008 1-7

50. J Ellenbeck, H Al-shatri, C Hartmann, Performance of decentralized interference coordination in the LTE uplink. in Proceedings of the IEEE 70th Vehicular Technology Conference, Anchorage, USA 20-23 September 2009 $1-5$

51. A Bou Saleh, Ö Bulakci, S Redana, B Raaf, J Hämäläinen, Impact of relay-torelay interference on the performance of LTE-Advanced relay networks. in Proceedings of the IEEE Wireless Communications and Networking Conference, Paris, France, 1-4 April 2011 1-5

52. A Bou Saleh, Ö Bulakci, S Redana, B Raaf, J Hämäläinen, A divide-andconquer approach to mitigate relay-to-relay interference. in Proceedings of the IEEE 22nd International Symposium on Personal, Indoor and Mobile Radio Communications, Toronto, Canada, 11-14 September 2011 1889-1893

53. J Góra, Interference mitigation for multi-carrier relay-enhanced networks. in Proceedings of the International Symposium on Wireless Communication Systems, Aachen, Germany, 6-9 November 2011 192-196

doi:10.1186/1687-1499-2012-124

Cite this article as: Góra and Redana: Resource management issues for multi-carrier relay-enhanced systems. EURASIP Journal on Wireless Communications and Networking 2012 2012:124.

\section{Submit your manuscript to a SpringerOpen ${ }^{\circ}$ journal and benefit from:}

- Convenient online submission

- Rigorous peer review

- Immediate publication on acceptance

- Open access: articles freely available online

- High visibility within the field

- Retaining the copyright to your article

Submit your next manuscript at $\gg$ springeropen.com 